\title{
La cientificidad de metodologías cuantitativa, cualitativa y emergentes
}

\author{
María Marcela Castañeda Mota
}

https://orcid.org/0000-0002-4847-4382, Universidad Veracruzana, México

Citar como: Castañeda, M. (2022). La cientificidad de metodologías cuantitativa, cualitativa y emergentes. Revista Digital de Investigación en Docencia Universitaria, 16(1), e1555. https://doi.org/10.19083/ridu.2022.1555

Recibido: 03/11/21. Revisado: 06/12/21. Aceptado: 14/01/22. Publicado: 25/02/22.

\section{Resumen}

Introducción: Se considera la aplicabilidad del modo de conocimiento científico para la resolución de problemas concretos de la vida cotidiana, individual o colectiva. Antecedentes: Desde el marco de criterios para la cientificidad se propone fundamentar el énfasis tradicional metodológico, con elementos teóricos y soportes epistémicos y lógicos, para justificar la aplicabilidad empírica orientada al bienestar social. Discusión: Se consideran elementos de contraposición y superación que derivan en propuestas con fundamento teórico para cumplir con objetivos cubiertos en la aplicabilidad de principios disciplinares; y con esto enmarcar metodologías tradicionales o emergentes.

Palabras Clave: métodos cuantitativos; métodos cualitativos; métodos emergentes; aplicabilidad.

\section{The scientificity of quantitative, qualitative and emerging methodologies}

\begin{abstract}
Introduction: It considers the applicability of the scientific mode of knowledge for the resolution of concrete problems of everyday life, individual or collective. Background: From the framework of criteria for scientificity, it is proposed to base the traditional methodological emphasis, with theoretical elements and epistemic and logical supports, to justify the empirical applicability oriented to social welfare. Discussion: Elements of contraposition and overcoming are considered that derive in proposals with theoretical foundation to fulfill objectives covered in the applicability of disciplinary principles; and with this, to frame traditional or emergent methodologies.
\end{abstract}

Keywords: epistemology; logical geography; methodologies; applicability.

\section{Introducción}

En este trabajo se presentan temas vinculados con el estudio de la dimensión científica para el planteamiento, realización y evaluación de programas que se implementen en el campo de la investigación psicológica aplicable; ya que, con la existencia de la diversidad de los caminos al conocimiento en la comprensión del mundo aparecen como declaraciones que permean el ámbito de la vida humana. Las implicaciones de supuestos teóricos consolidados han desarrollado gran parte de la historia de la cul- 
tura; por consiguiente, el científico asume su tarea de describir y explicar las regularidades del mundo regido por leyes naturales y portaciones empíricas (Bunge, 1985). No obstante, la aplicabilidad del modo científico a problemas concretos planteados para casos particulares o colectivos se encuentra en polémica en cuanto a sus propósitos: ante la interrogante de si la metodología científica toma en cuenta objetivos para el bienestar social.

Es así como resultan planteamientos de varios problemas de investigación que gozan en la actualidad de la acción planificada del método con carácter heurístico, orientados a una acción organizada y reflexiva hacia la sociedad (Dendaluce,1988). Esta condición permanece en cada demanda evolutiva que las instituciones sociales realizan a quienes generan el conocimiento, en especial al denominado conocimiento científico (Rorty, 1996). De tal forma, el propósito del estudio es presentar argumentos para el análisis y posible aplicación de principios derivados de la investigación hacia metodologías que consideren fundamentos para lograr la congruencia del terreno epistemológico, articulado con geografías lógicas pertinentes al estado de preguntas planteadas, con derivaciones y producción de datos que consoliden propuestas descriptivas o explicativas; considerando criterios fundamentales para la medición con propósitos de validación y valoración, teniendo en consideración su aplicabilidad en el terreno disciplinar y transdisciplinar en un contexto social. La psicología como ciencia natural, opina Ribes $(1982 ; 2004 ; 2018)$, debe abrir nuevos dominios empíricos y conceptuales para formalizar su importancia en el contexto social.

\section{Antecedentes}

Con relación a los elementos fundacionales de la cientificidad, el texto de Azcona (2013) considera que en la Edad Media se establecieron como principios de autoridad el verbalismo y uso del silogismo; conservando así el propósito de verdades últimas y trascendentes.

Para el siglo XVII, según Kothari (2004), se enfatizó en proponer reglas que conducían a un conocimiento válido; dichas reglas planteadas sobre todo en las obras de Descartes y Bacon, a pesar de que consideraban propósitos diferentes como el racionalismo y empirismo, ya identificaban coincidencias en privilegiar el desarrollo del método en el proceso de la investigación.

Así, en el inicio del conocimiento moderno y adheridos a las propuestas metodológicas de estos grandes filósofos, se consideró dividir el objeto de estudio de las disciplinas en componentes independientes y medibles; condiciones que podían compartir las ciencias naturales y sociales (Aranda, 1997).

En estas circunstancias, el tema de la medición era considerado como proceso permanente en la vida cotidiana y en la actividad del científico. Las operaciones fundamentales de una unidad para la medición se incorporaron como términos comunes en la derivación de los estudios. El perfeccionamiento de estos conceptos básicos es una de las mayores realizaciones humanas que proporciona los recursos necesarios de la cientificidad, el cambio tecnológico y la transformación social; sin embargo, es necesario incluir la discusión e implicaciones de la medición en el desarrollo del método (Kerlinger \& Lee, 2002).

Por consiguiente, en el estado contemporáneo de la metodología en la ciencia, se proponen pautas para analizar rutas fundamentales: a) características distintivas del objeto de estudio, b) lógica del proceso de investigación y c) supuestos filosóficos subyacentes. Estos supuestos filosóficos se abordan considerando tres tipos: 1) metodológicos, 2) ontológicos y 3) epistemológicos (Bunge, 1979). De esta manera, el método se considera el camino para dar la respuesta a la resolución del problema planteado, y dependiendo de una posición ontológica de la realidad, se permite la posibilidad de la decisión adecuada epistémica de su desarrollo para captar la riqueza y complejidad del fenómeno de estudio relacionado con el método (Bernal,2010, pp. 22-32).

Dicho en palabras de González (2002), la epistemología con relación al método es fundamental, y se define como el estudio filosófico de la ciencia que se considera como producto de dos orígenes: la filosofía y las disciplinas científicas particulares; indicando que la base filosófica de- 
riva de la teoría del conocimiento, la lógica y el materialismo histórico; mientras que las disciplinas particulares son la sociología y la historia de la ciencia. En el mismo texto de González se describen y exponen claramente tres períodos o etapas para el desarrollo histórico de la epistemología. El primer periodo se inicia en los tiempos griegos hasta las primeras décadas del siglo XX. Durante este período los estudios acerca de la epistemología fueron realizados por pensadores no especializados en la investigación de la ciencia. La segunda etapa se marca desde 1927 hasta los años 60; las aportaciones del Círculo de Viena y el neopositivismo fueron las más destacadas. En este período se identifica la especialización para estudiar la filosofía de la ciencia y es en1927 que se marca el nacimiento del empirismo lógico, considerado como la nueva epistemología. Es así como el neopositivismo se convierte en la filosofía dominante; permaneciendo vigente y con fuerza en la década de los años 60 influyendo en el método científico. En la tercera y última etapa se presenta la lucha de ideas entre los neopositivistas desplazados por los pospositivistas como Kuhn (1922-1996), Hanson (1924-1967), Feyerabend (1924-1994) y Toulmin (1922-2009); mientras que en Francia destacan Bachelard y Foucault. González (2002) afirma que para Carnap (1891-1970, positivista lógico), el conocimiento científico es una estructura racional que hace posible determinar si una hipótesis es apoyada lógicamente por la evidencia empírica y convoca a distinguir entre lo verdadero y falso; esto es, su carácter veritativo.

La oposición a esta perspectiva surge en la década de los 60 con autores como T. Kuhn, Feyerabend, Hanson, Tulmin. Los principales puntos que sostienen estos autores con respecto a la epistemología son: a) que la epistemología debe fundarse en el estudio de la historia de la ciencia y de los determinantes sociales del conocimiento científico; b) que la actividad científica no puede ser reducida al estudio de la cognición, ya que se deben incluir los fines y necesidades sociales a los cuales responde la ciencia; y c) que con el pospositivismo se destaca la importancia del sujeto y de los factores subjetivos en la ciencia y no solo la búsqueda de la objetividad como lo propone el empirismo lógico. El caso del no positivismo francés, radical y deliberado (representado por Bachelard, Canguilhem y Foucault) mantiene la oposición al positivismo por diversas rutas: Bachelard se inclina por la historia de las ciencias como recurso de análisis del conocimiento científico, mientras que para Foucault es la relación que hay entre saber y poder. Es el poder que posibilita los discursos y el saber científico o experto como producto de relaciones de poder. El mismo autor citado en esta sección hace referencia a una posición epistemológica denominada la psicología del reflejo creador, esto con base en las aportaciones de K. Marx (1818-1883). Esta propuesta epistemológica considera que la psiquis humana y el conocimiento científico son un reflejo de la realidad objetiva, pero también una creación subjetiva de sí mismo de la realidad percibida y pensada y de la cultura.

\section{La Participación del Quehacer Científico en Psicología}

Según la historia del proceso evolutivo del pensamiento cientìfico al interior de la psicología, la oposición al positivismo se manifiesta por los trabajos de Freud (1856-1939), Piaget (1896-1980), Maslow (1908-1970) y Rogers (1902-1987), entre otros. En este campo se presentan algunas derivaciones epistemológicas como el constructivismo, el construccionismo y la epistemología cualitativa. Para el constructivismo el conocimiento es una construcción del investigador y no es determinado de modo directo por los datos empíricos; niega el criterio de verdad que emerge de la verificación empírica. Por otro lado, el construccionismo social considera las conversaciones como la sustancia del mundo social, definidas como sistema de actividades conjuntas; para aceptar algo como verdadero, se requiere que los otros de la sociedad lleguen a la misma determinación: la ciencia resulta de la negociación entre actores en sus discursos interactivos, por consiguiente, la metodología llega a un gran dinamismo en búsqueda del conocimiento (Maletta,2009).

Con respecto a la epistemología marxista, sostiene González (2002), es determinada como una propuesta a mediados del siglo XIX con la segunda Tesis de Marx sobre Feuerbach, donde señala como problema, si al pensamiento humano se le 
puede atribuir una verdad objetiva y que sea considerado no como problema teórico, sino como problema práctico; entonces la discusión gira en torno a la objetividad y la subjetividad, entre la teoría y la práctica, lo individual y lo colectivo.

En este sentido, el positivismo afirma criterios tales como señalar que el conocimiento es objetivo, presentando como base los hechos dados por los sentidos exigiendo la exclusión de la interpretación subjetiva como determinante del conocimiento. Los hechos son la experiencia sensorial y su expresión verbal y la teoría cumple un papel de generalización de los hechos; propone la investigación cuantitativa y es el experimento su forma más acabada. Expresa una posición centrada en la ciencia y el método científico (Aranda, 1997). Ante estos argumentos, los antipositivistas rechazan el empirismo positivista y consideran que el conocimiento tiene como base la interpretación del sujeto (individual o colectivo); se niega la declaración de un conocimiento reflejo o copia de la realidad objetiva con existencia fuera de la conciencia; se declara que la elaboración teórica es una interpretación a partir de los hechos. Como diferencia, el antipositivismo se inclina por la investigación cualitativa, pero en algunos casos se puede conciliar con la investigación cuantitativa; este tipo de antipositivismo es una expresión del humanismo idealista, centrado en la problemática espiritual y social humana y así para González (2002), el humanismo científico considera el conocimiento como unidad de la teoría con la práctica, donde la teoría es una reflexión de la observación de los hechos acompañado de la práctica como ejercicio de verificación empírica. Es así como el conocimiento se considera objetivo y reflejo de la realidad objetiva que existe fuera de la conciencia. En el humanismo científico se incorpora la unidad dialéctica de la investigación cuantitativa con la cualitativa. De este modo, una primera conclusión es considerar el humanismo científico como elemento integrador y armonizador de pensamientos filosóficos contrapuestos, como son el positivismo y el antipositivismo. Los problemas epistemológicos que se abordan por las propuestas explicativas tienen relación con criterios de verdad y sostiene que la verdad está en la verificación empírica. Sin embargo, para el antipositivismo la verdad la proporciona la interpretación teórica y los hechos de la práctica. Específicamente para la escuela marxista el criterio de verdad se expresa en el reflejo creador, explicando que se considera un reflejo porque se basa en los hechos y se confirma con ellos. Se considera creador porque requiere una interpretación que no es dada directamente por los hechos, sino elaborada por la subjetividad del científico. Concluye diciendo que el investigador crea la hipótesis, crea la teoría, pero los hechos se encargan de verificarla o refutarla. Otro problema epistemológico vinculado está relacionado con la significación de la realidad; para este caso tienen coincidencia el positivismo y el antipositivismo, al negar la conciencia como generadora del conocimiento acerca de la realidad. En cambio, para Marx y Martí la ciencia puede conocer la realidad objetiva que existe fuera de la conciencia (De Gortari,1969).

Así mismo, González (2002), con relación al problema epistemológico de la realidad, describe que la realidad para la ciencia es la unidad de una parte de la realidad objetiva y por otra, su reflejo subjetivo. La ciencia no crea su objeto en cuanto realidad objetiva, sino que lo refleja, lo copia y crea su objeto en cuanto realidad para la ciencia. Se presenta la diferencia entre las llamadas ciencias naturales (explicativas) y las ciencias sociales (comprensivas). Con la comprensión se generan hipótesis, mientras que en las explicativas parten con hipótesis.

Para concluir, González (2002) plantea que el objeto material (y la realidad psíquica de otra persona) existen fuera del sujeto y se refleja en él (esto define el "conocimiento"). El mundo de las cosas determina la conciencia; y es la actividad con los objetos materiales los determinantes de la psiquis. Todo opera en el contexto de la interacción entre el ser y la conciencia social (entre la base y la superestructura). El neokantianismo reconoce que la misma realidad empírica puede ser considerada como naturaleza (universal, general, (método generalizador)) o historia (particular, individual (método individualizador). El positivismo se aferra a la investigación de lo general y al método cuantitativo, tanto para las ciencias sociales como para las naturales. Se presentan 
los puntos de confluencia y complementariedad en la investigación científica de la metodología cuantitativa y la cualitativa, manteniendo la unidad dialéctica. La propuesta final del autor es que se va de lo individual a lo general y viceversa, y esto es tanto para las ciencias naturales como para las sociales. Enfatiza la unidad dialéctica, en la base epistemológica de la investigación empírica (cuantitativa, cualitativa o emergente) donde en ese sentido el campo de la psicología ofrece importantes avances en el logro del conocimiento, ya que descubrir lo general y particular requiere de lo cuantitativo; si el propósito es revelar la naturaleza esencial, individual o colectiva, entonces la metodología cualitativa y mixta o emergente podría ser la indicada.

Considerando el método de las ciencias naturales y las sociales comparten similitudes: la unidad dialéctica de la interpretación teórica con la verificación empírica de lo individual a lo general. Sin embargo, se deberá tener en cuenta que en las ciencias sociales se pondera la vivencia, la comunicación, la introspección y la autoobservación (Rojas,1982).

\section{Derivaciones en Rutas de Combinación o Integración Metodológica}

Como resultado de las condiciones señaladas, se aprecia que al interior de las ciencias existe consenso sobre el objeto diverso que define el conocimiento en general y de la psicología en particular. La presencia de estas divergencias se da en los planteamientos de cómo examinar un campo particular del fenómeno investigado, generando metodologías considerando por consiguiente la cuantitativa, cualitativa y mixta (Hernández \& Mendoza, 2018). Respecto al campo de estudio de la psicología como ciencia de múltiples paradigmas distintos entre sí, que además transitan por rutas independientes, paralelas o divergentes, existe un desacuerdo hasta el momento sobre el objeto de conocimiento específico abordado. El establecimiento del objeto de estudio tiene dos implicaciones: la definición ontológica y el compromiso epistémico del mismo objeto de estudio y en el caso de la psicología, debe guardar congruencia en cada propuesta de investigación al analizar fenómenos de distinta naturaleza y a partir de esto, los métodos, las medidas, criterios de evidencia y explicaciones pueden ser utilizados (Ribes, 2000).

De tal forma, Azcona (2013) indica que las investigaciones cuantitativas se desarrollan siguiendo la lógica del método hipotético-deductivo, basado en un ciclo de inducción, deducción e inducción; mientras que lo cualitativo antepone en los propósitos metodológicos incorporar incidencias socio-históricas que corresponden a ideologías de los estados. La anterior condición compromete las hipótesis a la universalidad y trascendencia. Desde la perspectiva metodológica, se distinguen dos grupos que comparten un enfoque experimental: uno con propósitos de hallar causalidad y el otro centrado en correlaciones (Buendía \& Hernández, 1995). La propuesta cuantitativa tiene fundamento en un realismo dividido en realismo ontogénico y realismo epistemológico, realismo ontológico que plantea aquello que se conoce existe y es cognoscible (Bunge,1999).

Mientras que en el método cualitativo se hace referencia, cita Martínez (1998), a una multiplicidad de estudios que tienen como factor común el rechazo a lo cuantitativo; enfatiza como principal preocupación el estudio de la subjetividad y coloca en el marco de la complejidad al constructivismo y la hermenéutica; el privilegio que realiza es hacia la introspección con todos sus ingredientes internalistas: comprensión, empatía, motivación, finalidad, entre otros y la lógica de lo cualitativo es de tipo inductivo como proceso recursivo (iterativo). En opinión de Sandoval (1996), el dato recolectado pertenece al rubro de las significaciones, derivado de la interacción con los participantes, manejando el muestreo no aleatorio; de esta forma las variables no sufren manipulación experimental y se consideran patrones culturales propios y las relaciones de significación son reconstruidas por medio de narraciones y no por índices estadísticos. Se exponen múltiples caminos para construir el conocimiento y se muestra la imposibilidad de establecer principios racionales y universales; el objetivo es conocer el significado de su interpretación (Escudero \& Cortez, 2018).

Por otro lado, lo cuantitativo - señala Azcona (2013) - es sintético, holístico, local y cualificador. La realidad en ciencias humanas se ajusta 
mejor a la idea de sistemas. De este modo, la Teoría General de los Sistemas se constituye como metateoría para la transdisciplinariedad. La concepción ontológica del sistemismo se denomina complejidad donde se considera el desorden, lo aleatorio, la incertidumbre, lo recursivo, lo paradójico. Por tales motivos, el enfoque mixto no es una mezcla de lo cuantitativo con lo cualitativo; se propone como enfoque autónomo derivado, pero no dependiente de lo cuantitativo y lo cualitativo. Se basa en una figura metodológica propia de la geometría, denominada triangulación y hace depender la existencia de los objetos a partir de los conceptos; y como los conceptos los piensa el individuo, entonces la explicación de la realidad es una construcción de los individuos (Wartofsky, 1987). Para reforzar esta perspectiva se integran recursos y procedimientos del ámbito cuantitativo y cualitativo (Ragin, 2007).

Debido al énfasis actual que considera al Método Científico como central para el quehacer de la ciencia, Bernal (2010, pp. 22-32) propone justificar la importancia de elementos epistemológicos para superar la pregunta relativa al caso de si la filosofía tiene un método único. El autor presenta un análisis conceptual del término "epistemología”. Inicia su argumento con el contexto tradicional formal de raíces aristotélicas y presenta una taxonomía categorial de los tipos de ejercicios epistemológicos que tienen como referente la ubicación de la práctica de la filosofía de la ciencia, propósitos y consecuencias; donde se mantiene el carácter descriptivo, mas no justificatorio. Para el autor, el ejercicio de la epistemología actual, que denomina regional, exhibe requerimientos específicos para cada disciplina. Por lo que pretende alejarse del énfasis metodológico para exponer la importancia de lo formal. Se retoma la singularidad del término epistemología, que dada la justificación previa debería presentarla como "de las epistemologías" y las examina como problemas que son propios para el ejercicio epistemológico. Para esto utiliza una mezcla de vaguedades filosóficas y términos pretendidamente respaldados por una perspectiva constructivista. De este modo concluye invocando racionalidad en el manejo de los argumentos y una “actitud científica”, pero no clarifica el término.
En el capítulo sobre el concepto de ciencia social, Bernal (2010, pp. 34-47), realiza una cronología de la sociedad para identificar que la ciencia se ha conceptualizado con diferentes enfoques y la derivación de una pluralidad metodológica. Este es el modo de presentar sus datos; sin embargo, no expone criterios que determinen la clasificación histórica que realiza. Propone a la hermenéutica como opción para la búsqueda de independencia del positivismo o para confrontarlo e insertar un sesgo hacia la desvalorización de las ciencias sociales. En consecuencia, el planteamiento se puede interpretar como la ideología del llamado movimiento neopositivista. El autor citado expone una conclusión parcial no fundamentada, y deja sin aclarar cómo el bienestar se convierte en el objeto de estudio de la teoría en las ciencias sociales.

Cuando Bernal (2010, pp. 48-55) aborda el tema de la complejidad y la ciencia, menciona que la complejidad se organiza como método (o pensamiento complejo), cosmovisión o como ciencia. Derivado del paradigma de la complejidad, en la ciencia se presentan cambios y se habla de lo polidisciplinario o multidisciplinario, interdisciplinario y transdisciplinario. Implicando que la complejidad interpreta la realidad como pluridimensional, compleja, paradójica, cambiante, con orden y desorden, con logros y frustraciones. Participar del compromiso paradigmático de la complejidad tiene como derivación adoptar un enfoque interdisciplinario en la construcción del conocimiento científico; así como integrar el conocimiento científico con otros tipos de conocimiento, con el propósito de acercarse a la realidad, aceptando que este acercamiento no es definitivo de la realidad. Esta forma de interpretar la complejidad comparte argumentos asociados con el escepticismo; argumentos que extienden sus efectos a todo aquello que emana como sucedáneo de la complejidad, o con el surgimiento de nuevas categorías. El autor finaliza proponiendo un retorno al orden, la unidad, la integración y el intercambio; elementos que siempre han caracterizado las epistemologías que sustentan los diversos modos de conocer.

Con Aranda (1997) se plantea que el denominado pensamiento posmoderno, al proponer tras- 
cender en nuevas rutas metodológicas, critica desde el historicismo y la filosofía de la ciencia, a la epistemología moderna, desde ángulos relacionados con el sujeto que conoce y de aquello constituido como objeto de conocimiento; la crítica se extiende hacia la semántica de términos esenciales y a las narrativas globales. En este pensamiento posmoderno reconoce a Gastón Bachelard (1884-1962) como fundador del análisis y metodología estructurales. El análisis estructural enfatiza la importancia de la epistemología en la práctica de la ciencia y determina como bases metafísicas de la ciencia al racionalismo y al realismo. Otra propuesta es considerar la característica evolutiva en la historia de la ciencia al señalar el carácter discontinuo en relación a explicaciones previas; de aquí se deriva el concepto de ruptura del horizonte epistemológico. Señalando que la ciencia realiza interpretaciones de relaciones y no en términos sustantivos: describir los hechos en la forma más simple. Con estos argumentos se propone el término suprarrealismo como enriquecimiento del racionalismo por medio de relación referencial con el mundo material. Otra aportación de Bachelard, señalada por Kumar (2010) tiene que ver con la negación del énfasis que pone a la percepción como punto de inicio de la epistemología posmoderna; en su lugar propone a la imaginación creativa como actividad de la voluntad del sujeto. Esta imaginación creativa se distingue de los conceptos, los cuales son una traducción del mundo exterior en conceptos. Volviendo con Aranda, indica que otro autor ubicado en el pensamiento posmoderno es Michel Foucault (1926-1984) que discute sobre el método de la historia y considera evitar la proyección de significados en la articulación de la historia. Y señala que Foucault, trata a la causalidad histórica como sospechosa: para él solo existen los efectos y actos materiales; en consecuencia, no hay un significado esencial en la historia, ni tema detrás de la acción, como tampoco hay un orden esencial en la historia; el orden resulta del propio acto de escribir la historia, y además describe que Foucault concluye mencionando que las practicas devienen de modos de pensar; cada uno con su propia lógica, estrategia, evidencia y razón. Para este autor, la historia se escribe desde la perspectiva del presente; acepta que el presente siempre está en curso de transformarse, con esta convicción, el pasado adquiere nuevos significados a la luz de los actos recientes. La historia es siempre una historia del presente. La historia de los marcos teóricos del conocimiento y los modos de comprensión están en cambio perpetuo. Es la epistemología que estudia estos cambios que para Foucault es la gramática de la producción del conocimiento que se expresa por la práctica de la ciencia, la filosofía, el arte y la literatura. Con la epistemología se asocian los actos materiales con el pensamiento y las ideas.

George Canguilhem (1904-1995), citado también por Aranda (1997) como otro contribuyente del pensamiento posmoderno francés. Este autor se rebela ante la epistemología moderna, pues considera que la ciencia establecida es aquella que determina la escuela positivista, por lo que se enfrenta a los elementos fenomenológicos y esencialistas que dan fundamento al positivismo, y se desplaza hacia una nueva forma de explicación que considera el conocimiento como resultado de relaciones y diferencias. Así, para Canguilhem, la historia de la ciencia ya no es la manifestación de una mente, sino que parte de configuraciones epistemológicas para construir su propio marco intelectual, es lo que denomina historiografía estructuralista. Canguilhem refiere que la historia de la ciencia se había considerado como una ilusión retrospectiva (el pasado conduce hacia el presente), donde el presente se conceptualiza estático e inmutable, luego entonces la historia de la ciencia que se escribe permanecerá válida en el futuro. Sin embargo, Canguilhem como Bachelard sostienen que la ciencia es dinámicamente abierta y discontinua y no cerrada y continua; por ese motivo, un hallazgo menor en el presente se vuelve importante frente a un nuevo problema. La ciencia es una estructura que hace y rehace su historia a cada instante: el conocimiento depende estrechamente del medio circundante; la verdad y el error solo valen dentro de un contexto histórico en particular, que cambia según el punto de vista (Sierra, 2013).

Por consiguiente, Aranda posiciona a Michel Serres (1930-2019) como filósofo de la invención, donde se incluyen las transformaciones como tra- 
ducción, comunicación y metáfora, describe que para Serres, la naturaleza del conocimiento constituye un reto a la homogeneidad; las ciencias son interrelacionadas incluyendo el arte y Serres toma el modelo de comunicación para hacer una analogía con el conocimiento y propone un componente para el cual considera importante: el ruido, ya que las teorías formales excluyen el ruido, el ruido es consustancial tanto en el lenguaje como en los dispositivos de comunicación; proponiendo Serres al análisis estructural como método comparativo para navegar entre dominios y realidades diferentes. En este sentido, la ciencia puede mantener su viabilidad en la medida que incorpora el arte en sus diferentes expresiones, pues el arte da oportunidad a la inspiración de lo impredecible.

El autor referenciado por Aranda (1997) como representante de la posmodernidad es JeanFrançois Lyotard (1924-1998). Este autor centra la atención en el concepto de metanarrativa, la cual consiste en otorgar un propósito creíble para las acciones de la ciencia o de la sociedad. La posguerra hace menos posible las grandes narrativas de la ciencia (conocer por el conocimiento mismo y el conocimiento como emancipación del oscurantismo). Las tecnologías desplazan a la ciencia hacia un propósito de los propios medios para lograr la acción. Para Lyotard, el sentido de la ciencia es un juego de lenguaje; las reglas de la ciencia son inmanentes a su propio juego. A pesar de esto, Lyotard señala que la tecnología sigue el principio de rendimiento óptimo (performatividad): máximas salidas a partir de mínimas entradas, riqueza, eficiencia y verdad.

Como opina Calero (2000), en el campo de las ciencias sociales se presentan condiciones epistémicas y metodológicas no cubiertas de modo explícito. El autor identifica dos posibilidades de cientificidad para las humanidades, una ubicada como continuidad y emulación de los objetivos de las ciencias naturales y la otra reclamando autonomía y criterios propios para definirse como ciencia. Como antecedente en la historiografía científica de las ciencias sociales, se mencionan orígenes cualitativos, y fue lo cuantitativo quien tuvo que justificarse. El enfoque cuantitativo evolucionó y se fortaleció tanto que llegó a dominar el escenario de las ciencias naturales y sociales.
Actualmente. señala Azcona (2013), lo cualitativo renace de su aislamiento y se convierte en oposición a la cual se exige justificación. Además de la confrontación por cuanto al objeto y método, la lucha incorpora el problema de la generación de conocimientos como práctica social determinada y mediada por el lenguaje. No se determina abiertamente como metodología mixta a los intentos de mezclar lo cualitativo con lo cuantitativo, alejándose más del criterio de considerar un marco teórico. Con respecto a la postura mixta, dentro de la sociología, se identifica a los puristas (que se oponen a la mezcla); los eclécticos (ambos enfoques son válidos) y los pragmáticos (que articulan los métodos, dependiendo del tema estudiado). Otra forma de integrar lo cuantitativo con lo cualitativo se define por el objetivo del estudio; si el objetivo es teórico se va por la ruta tradicional, si no lo es, entonces la opción sería un análisis marxista, de este modo, la teoría y la práctica en las ciencias sociales constituyen dos momentos de un solo proceso denominado investigación científica; aparte se identifica el problema ético para cada tipo de método: lo cuantitativo se sirve de los participantes mientras que lo cualitativo se propone la comunicación con los sujetos del estudio. Asunto importante lo constituye la validez externa o generalización; muy alta en lo cuantitativo y baja en lo cualitativo (Hernández, 2007). Esto parece referir a distinguir entre generalización y confiabilidad; con el sustento de que la generalización se da para el plano teórico respecto del diseño, mientras que la confiabilidad se enfoca en los datos, en el contexto de un método estadístico Desde aquí se observa la importancia del regreso a lo empírico en la vida cotidiana; propuesta similar que se realiza desde una perspectiva marxista al sugerir el mismo tipo de constatación en investigación cuantitativa. Al interior de las ciencias humanas (sea que requiere seguir la ruta de las ciencias naturales o una definición específica con teorías y métodos propios) el uso de uno u otro proceder, depende del momento que al investigador le interesa privilegiar y del tema que se elige para investigar con una verdadera transdisciplinariedad (Roca, 1993).

En consecuencia, la ciencia debe mantener una perspectiva de aplicabilidad en su método, debe 
considerar al individuo como parte de una sociedad, como representante de las interacciones que se establecen en circunstancias, tiempo y espacio determinados dentro de este sistema social. En este sentido Wolff (1976) indica que para la pertinencia en la aplicación, en cuanto a la disciplina psicológica se debe tomar en cuenta: la significación social de las metas establecidas en lo que se pretende aplicar, la justificación social de los procedimientos empleados y la importancia social de los efectos obtenidos. De manera que el método que establezca la pertinencia de las acciones sociales que se estén empleando se hará a partir de la determinación empírica de los criterios de validación social. Es decir, que la validación social de la psicología aplicada se debe dar a través de la explicitación y análisis crítico de los determinantes sociales que enmarcan a la situación donde se da el comportamiento, considerando los criterios en el contexto de su valor de uso social real. Para reconocer la valoración social como un indicador decisivo dentro de la disciplina, es indispensable identificar la existencia de una doble dimensión en el comportamiento humano, una relacionada con las prácticas individuales, las cuales están condicionadas a circunstancias (históricas, culturales, sociales, etc.) que en dicha práctica se originan y se mantienen como interacción social. Y la otra, la existencia de la relación social solo como prácticas individuales vinculadas por condiciones comunes en tiempo y espacio.

Es así como el ser humano se comporta dentro de una sociedad a través de interacciones, con sujetos y objetos que están a su alrededor, es por eso que no se puede entender un comportamiento humano como algo aislado e independiente, sino como parte de un sistema, donde su comportamiento tiene sentido dentro de una sociedad a partir de un sistema de interacciones. Por consiguiente, lo social y lo individual no constituyen, en el caso del comportamiento humano, dimensiones contrarias, ya que expresan diversos niveles de un mismo conjunto complejo de relaciones y resulta importante apuntar que los problemas generados dentro de esta disciplina científica trascienden el aspecto puramente técnico de la misma, es decir que se define como una problemática con validez social, la cual legitima las ca- racterísticas y circunstancias del comportamiento del sujeto. Desde este análisis del problema definido en lo social e ideológico surgirá consecuentemente un análisis aplicado que hará contacto con los problemas sociales, con la intención de brindar soluciones en el aspecto individual sin perder de vista que tanto la teoría y el método deben estar sujetos al contexto colectivo en el que se encuentra (Ribes, 2000).

Finalmente, existe un objeto general de conocimiento y divergencias en los planteamientos de cómo examinar un campo particular del fenómeno investigado. Bajo esta perspectiva, en el campo específico de la psicología como ciencia que postula varias "psicologías", distintas entre sí, que transitan por rutas independientes, paralelas o divergentes. Esta condición reside en el desacuerdo existente sobre el objeto de conocimiento específico abordado. El establecimiento del objeto de estudio tiene dos implicaciones como se ha mencionado: la definición ontológica y el compromiso epistémico del objeto de estudio y al discutir el caso de la psicología, se debe considerar que cada propuesta analiza fenómenos de distinta naturaleza y a partir de esto, los métodos, las medidas, criterios de evidencia y explicaciones deben converger armónicamente (Ribes, 2000).

A pesar de ello la psicología, como creadora de conocimiento validado en condiciones específicas, se ha consolidado como modo de conocimiento científico en varias de sus aportaciones, con sentido pragmático formando parte de los problemas sociales, ya que es evidente que las tecnologías tienen un peso social considerable. En este sentido, es importante partir de la práctica individual para identificar la concepción social, reconociendo a la sociedad como sistema de relaciones con un nivel de existencia de lo individual (Ribes, 2018).

\section{La Aplicabilidad Posible de la Investigación Psicológica}

En relación con la aplicabilidad de principios derivados de la investigación científica, en especial del análisis funcional de la conducta, Ribes 
(2009) identifica cinco etapas de seguimiento en este proceso. Primero se hace un recorrido en el mundo del conocimiento ordinario, compartido socialmente por todos los individuos. Posteriormente, se expone como historia natural donde se buscan las regularidades en las formas de interacción cotidiana. En la tercera etapa, a partir del lenguaje técnico, se describen las formas de las distintas regularidades observadas y promueve la visibilidad de nuevas regularidades; dichas descripciones se realizan en un lenguaje abstracto que no hace referencia a particulares; finalmente, se retorna de la teoría hacia el mundo cotidiano. Es decir, se usan categorías de un cuerpo teórico para interpretar un conjunto de observaciones de otro campo disciplinario (multidisciplinaria).

Con estos antecedentes se puede afirmar que toda aplicabilidad de principios psicológicos tiene carácter valorativo, donde lo psicológico se encuentra en la dimensión individual de la relación social a la que pertenece; es decir, que el individuo es la representación de dichas prácticas sociales, es así cómo la dimensión psicológica se encuentra inmersa en toda problemática social, la cual no existe de forma per se, sino que reside en un acto de valoración. En este sentido, Ribes (2009) ratifica que todo acto de comportamiento tiene sentido solo en términos del contexto social en que tiene lugar, es decir que dicho acto es un acto de valoración del y en el contexto en el que ocurre. El carácter valorativo de dicho problema hace referencia al hecho de apreciar un acto como problemático, es decir es calificado dependiente de a quién afecte y quién lo valore. No es un hecho que está ahí como objeto palpable en la realidad o como algo determinado por su existencia. Donde la especificación del problema deberá ser "reconstruida" entre el psicólogo y el usuario, con el propósito de satisfacer dos requerimientos: 1) por un lado poder describir la naturaleza funcional del problema, analizando al individuo y las prácticas en las que está inmerso en circunstancias determinadas y 2) identificar la relación de las circunstancias donde se genera dicho problema en condiciones similares (Ribes \& López, 1985).

\section{Discusión}

Considerando el vínculo del quehacer científico y la aplicación a problemas concretos de la vida cotidiana, se proponen la filosofía de la ciencia y la psicología como elementos conjuntos para observar el ejercicio orientado a la aplicación del método. Se propone que esta tarea inicie desde el interior de las disciplinas científicas como reflexión metateórica, sin olvidar el papel legitimador de relaciones de poder (Castorina, 2016). El mismo autor sugiere la independencia de la práctica psicológica para constituirse como disciplina y explica la influencia de modelos representacionales junto a otros factores, en especial los relacionados con el ejercicio de los métodos de investigación utilizados. Señala el autor citado que los psicólogos y/o los psicopedagogos no estudian un niño "natural"; con independencia de lo que hacen con él los educadores y los sistemas médicos, las prácticas pedagógicas y médicas se consideran ejercicios que parten de un dispositivo de normativización adjudicado a la infancia. Se presentan justificaciones y ejemplos de las expresiones del ejercicio de la metodología que se encuentran entre los telones de la actividad científica y las reflexiones epistemológicas. Esto quiere decir, desde el punto de vista epistemológico, que las teorías psicológicas originadas fuera del mundo social y han sido aplicadas sin considerar la especificidad de este campo (representacionales), los procesos entonces estudiados se encuentran separados del mundo y la cultura a la persona en situación o los individuos activos hacia su cultura.

Como alternativa, Castorina (2016) propone el cuestionamiento de las condiciones sociales en la legitimación del ejercicio de ese poder de la sociedad, además de la participación del científico y del profesional que aplica. El marco de esta propuesta para el análisis de las condiciones sociales es la figura de Marco Epistémico (ME), como concepción de mundo que se asume de manera silenciosa, y que interviene sobre los distintos niveles de las prácticas y los procesos de investigación. Se trata de una serie de creencias filosóficas marcadas por la cultura y las relaciones de poder en una sociedad histórica. El marco epistémico 
se encuadra dentro de la metodología dialéctica; como lógica empírica y no ideológica; de este modo, se propone superar la disociación dualista por un modelo articulatorio de los diversos componentes de los hechos. Esto es, se acepta superar la perspectiva de objetos aislados que conforman los hechos, para colocar el énfasis en las relaciones que se establecen entre ellos; con esta perspectiva se analizan los procesos que se observan tanto en la generación del conocimiento como en la aplicación de las metodologías.

\section{Referencias}

Aranda, A. (1997). La crítica posmoderna de la ciencia: Una genealogía francesa. Ciencia ergo sum, 4(2), 223-229.

Azcona, M. (2013). Contexto onto-epistemológico de las investigaciones científicas. En M. J. Sánchez (Coord.), Investigar en ciencias humanas. Reflexiones epistemológicas, metodológicas y éticas aplicadas a la investigación en Psicología (pp. 44-95). Universidad Nacional de La Plata.

Bernal, C. A. (2010). Epistemología o filosofía de la ciencia. En Autor (Ed.) Metodología de la Investigación. Administración, economía, humanidades y ciencias sociales ( $3^{\mathrm{a}}$ ed., pp. 22-32). Pearson Educación.

Bernal, C. A. (2010). Sobre el concepto de ciencia social. En Autor (Ed.), Metodología de la Investigación. Administración, economía, humanidades y ciencias sociales ( $3^{\mathrm{a}}$ ed., pp. 34-47). Pearson Educación.

Bernal, C. A. (2010). Complejidad e interdisciplinariedad de la ciencia. En Autor (Ed.) Metodología de la Investigación. Administración, economía, humanidades y ciencias sociales ( $3^{a}$ ed., pp. 48-55). Pearson Educación.

Buendía, C., \& Hernández, S. (1995). Métodos de la Investigación en Psicopedagogía. Mc Graw Hill.

Bunge, M. (1979). La Investigación científica. Ariel.

Bunge, M. (1985). Racionalidad y Realismo. Alianza Editorial.

Bunge, M. (1999). Las Ciencias Sociales en discusión: una perspectiva filosófica. SudAmericana.

Calero, J. L. (2000). Investigación cualitativa y cuantitativa. Problemas no resueltos en los debates actuales. Rev Cubana Endocrinol, 11(13), 192-198.

Castorina, J.A. (2016). Algunos problemas epistemológicos de la teoría psicológica y de la práctica psicopedagógica. Revista Pilquen, 13(2), 48-62.

De Gortari, E. (1969). 7 ensayos filosóficos sobre la ciencia moderna. Grijalbo.
Dendaluce, I.. (1988). Aspectos metodológicos de la investigación educativa. Narcea

Escudero, C. L., \& Cortez, L. A. (Coord.). (2018). Técnicas y Métodos Cualitativos para la Investigación Científica. UTMACH.

González, D. J. (2002). Epistemología y Psicología: Positivismo, antipositivismo y Marxismo. Revista Cubana de Psicología, 19(2), 150-159.

Hernández, H. R. (2007). Métodos de investigación en Psicopedagogía. McGraw-Hill.

Hernández, H. R., \& Mendoza, C. P. (2018). Metodología de la investigación. Las rutas cuantitativa, cualitativa y mixta. McGraw-Hill.

Kerlinger, F.N., \& Lee, H.B. (2002). Fundamentos de medición. En Autores, Investigación del comportamiento. Métodos de Investigación en ciencias sociales (4 ${ }^{\mathrm{a}}$ ed., pp. 565-580). McGraw Hill.

Kothari, C. R. (2004). Research methodology: Methods and techniques. New Age International.

Kumar, R. (2010). Research Methodology: A Step-by-Step Guide for Beginners. Sage Publishers

Maletta, H. (2009). Epistemología Aplicada: Metodología y Técnica de la producción científica. Centro Peruano de Estudios Sociales. Universidad del Pacífico.

Martínez, M. (1998). Epistemología y Metodología Cualitativa en las Ciencias Sociales. Trillas.

Ragin, C. (2007). La construcción de la investigación social. Introducción a los métodos y su diversidad. Siglo del Hombre editores - Universidad de los Andes.

Ribes, E. (1982). El conductismo: reflexiones críticas. Fontanela

Ribes, E. (2000). Las psicologías y la definición de sus objetos de conocimiento. Revista Mexicana de Análisis de la Conducta, 26(3), 367-383.

Ribes, E. (2004). Acerca de las funciones psicológicas: un post-scriptum. Acta Comportamentalia, 12(2), 117 127.

Ribes, E. (2009). La psicología como ciencia básica. ¿Cuál es su universo de investigación? Revista Mexicana de Investigación en Psicología, 1(2), 7-19.

Ribes, E. (2018). Transitando entre los límites: lo psicológico en la multidisciplina y en la interdisciplina. En Autor, El estudio científico de la conducta individual: Una introducción a la teoría de la Psicología (pp. 367-424). Manual Moderno.

Ribes, E., \& López, F. (1985). Teoría de la Conducta: Un análisis de campo y paramétrico. Trillas.

Roca, J. (1993). Lenguaje y ciencia psicológica. Acta Comportamentalia, 1(1), 27-38.

Rojas, R. (1982). Guía para realizar investigaciones sociales. Universidad Nacional Autónoma de México.

Rorty, R. (1996). Contingencia, ironía y solidaridad (Reimp.). Ibérica.

Sandoval, C. (1996). Investigación Cualitativa (Vol.4). ICFES Asociación Colombiana de Universidades e Instituciones Universitarias Privadas. 
Sierra, E. (2003). Nuevos elementos para la reflexión metodológica en sociología. Del debate cuantitativo/ cualitativo al dato complejo. Papers: Revista de sociología, 70(12), 57-81.

Wartofsky, M. W. (1987). Introducción a la filosofía de la ciencia. ( $\left.3^{\mathrm{a}} \mathrm{ed}\right)$. Alianza.
Wolff, M. M. (septiembre, 1976). Social Validity: The case for subjective measurement or how applied behavior analysis is finding his heart [Sesión de conferencia], Asociación Psicológica Americana, Washington, D.C.

RIDU / Revista Digital de Investigación en Docencia Universitaria / e-ISSN: 2223-2516

(c) Los autores. Este artículo es publicado por la Revista Digital de Investigación en Docencia Universitaria del Área de Institutional Research and Effectiveness de la Dirección de Aseguramiento de la Calidad, Universidad Peruana de Ciencias Aplicadas. . "Este es un artículo de acceso abierto, distribuido bajo los términos de la LicenciaCreativeCommons Atribución 4.0 Internacional (CC BY 4.0) (https://creativecommons.org/licenses/by/4.0/deed.es), que permite el uso, distribución y reproducción en cualquier medio, siempre que la obra original sea debidamente citada." 